\title{
Efecto de la suplementación de progesterona y enfriamiento artificial post-inseminación sobre la eficiencia reproductiva de vaquillas Holstein durante el verano
}

\author{
Effect of progesterone supplementation and artificial cooling post-insemination on reproductive \\ performance of Holstein heifers during summer
}

\author{
A Correa-Calderóna ${ }^{\mathrm{a}}$ CI Gómez-Álvarez ${ }^{\mathrm{a}}$, L Avendaño-Reyes ${ }^{\mathrm{a}} \mathrm{R}$ Diaz-Molina ${ }^{\mathrm{b}}$, \\ S Medina-Cervantes ${ }^{\mathrm{a}}$, F Rivera-Acuña ${ }^{\mathrm{c}}$, P Luna-Nevárez ${ }^{\mathrm{c}}$, Ủ Macias-Cruz ${ }^{\mathrm{a}^{*}}$ \\ a Instituto de Ciencias Agrícolas, Universidad Autónoma de Baja California, Mexicali, Baja California, México. \\ ${ }^{\text {b }}$ Facultad de Medicina, Universidad Autónoma de Baja California, Mexicali, Baja California, México. \\ ${ }^{\mathrm{c}}$ Departamento de Ciencias Agronómicas y Veterinarias, Instituto Tecnológico de Sonora, Ciudad Obregón, Sonora, México.
}

\begin{abstract}
SUMMARY
A total of 137 Holstein heifers $(\mathrm{BW}=350 \pm 24 \mathrm{Kg}$ ) were used to assess the supplementation of progesterone plus artificial cooling after artificial insemination (AI) on conception rate (CR) and physiological responses during the summer time. Heifers were randomly assigned into three treatments: 1) Control treatment ( $\left.\mathrm{T}_{1}\right)$, only with shade, visual heat detection and AI according to AM-PM rule (n $=45)$; a second treatment $\left(T_{2}\right)$ with the same reproductive management of $T_{1}$, but receiving $21 \mathrm{~d}$ of artificial cooling after AI $(n=46)$; and a third treatment $\left(\mathrm{T}_{3}\right)$ with heifers under timed AI plus progesterone supplementation ( $\mathrm{d}$ 4-14 after AI), and a cooling period similar to $\mathrm{T}_{2}(\mathrm{n}=46)$. The maximum average of THI during the study was 86 , while the minimum was 78 . Blood samples were collected at days 5,9 y 14 post-AI to determine progesterone concentration, respiration rate (17:00 h), rectal temperature $(18: 00 \mathrm{~h})$, while vaginal temperature was recorded every 15 min during $24 \mathrm{~h}$ twice a week. These variables measured over time were analyzed with a repeated measurement by using the REPEATED and RANDOM statements of the MIXED procedure of SAS. Conception rate was analyzed with a chi-squared test. Respiration rate was different $(\mathrm{P}<0.05)$ among treatments $\left(\mathrm{T}_{1}: 89.0 \pm 6.5 ; \mathrm{T}_{2}: 80.0 \pm 6.5 ; \mathrm{T}_{3}: 83.0 \pm 6.5\right.$ breaths/ $\mathrm{min}$ ), while the vaginal temperature of $\mathrm{T}_{1}$ was higher $(\mathrm{P}<0.05)$ than $\mathrm{T}_{2}$ and $\mathrm{T}_{3}$ only from 10:00 to 17:00 h. The rectal temperature, $\mathrm{CR}$ and levels of $\mathrm{P}_{4}$ were similar $(\mathrm{P}>0.05)$ among treatments. The supplementation progesterone and artificial cooling after AI did not show any improvement on conception rate.
\end{abstract}

Key words: dairy heifers, conception rate, artificial cooling, progesterone.

\section{RESUMEN}

El objetivo del estudio fue evaluar la suplementación de progesterona y enfriamiento artificial de los animales, post inseminación artificial (IA) sobre la tasa de concepción (TC) y respuestas fisiológicas en vaquillas Holstein durante el verano. Ciento treinta y siete vaquillas con un peso promedio de $350 \pm 24 \mathrm{~kg}$ fueron distribuidas al azar a uno de tres tratamientos: tratamiento testigo ( $\mathrm{T}_{1}$ ), vaquillas beneficiadas con sólo sombra en la parte central del corral, detección visual del celo e inseminación artificial (IA) de acuerdo a la regla AM-PM $(n=45)$; segundo tratamiento $\left(T_{2}\right)$, vaquillas bajo el mismo manejo reproductivo que $T_{1}$, más un período de enfriamiento artificial de 21 d post-IA $(n=46)$; tercer tratamiento $\left(T_{3}\right)$, vaquillas bajo un protocolo de inseminación a tiempo fijo más suplementación de $\mathrm{P}_{4}$ (día 4-14 post-IA) y enfriamiento artificial al igual que $\mathrm{T}_{2}(\mathrm{n}=46)$. Durante el experimento el promedio máximo de THI fue 86 en tanto que el mínimo fue 78. La FR, TR, TV y $\mathrm{P}_{4}$ fueron analizadas por medio de un modelo de medidas repetidas en el tiempo, mientras que la TC se analizó por chi-cuadrado. La FR difirió $(\mathrm{P}<0,05)$ entre los 3 tratamientos $\left(\mathrm{T}_{1}: 89,0 \pm 6,5 ; \mathrm{T}_{2}: 80,0 \pm 6,5 ; \mathrm{T}_{3}: 83,0 \pm 6,5 \mathrm{r}\right.$ espiraciones/min), mientras que las TV registradas durante las 24 horas del día solo difirieron $(\mathrm{P}<0,05)$ entre el grupo testigo y los tratamientos $\mathrm{T}_{2} \mathrm{y} \mathrm{T}_{3}$ de las 10:00 a las 17:00. La TR, TC y los niveles de $\mathrm{P}_{4}$ fueron similares entre tratamientos $(\mathrm{P}>0,05)$. La suplementación de progesterona combinada con enfriamiento artificial post-IA no mejoró la TC con respecto al grupo testigo.

Palabras clave: vaquillas lecheras, tasa de concepción, enfriamiento artificial, progesterona.

Aceptado: 17.10.2013

* Carretera a Delta S/N, Ejido Nuevo León, Mexicali, B.C, C.P 21705, México; Ulisesmacias1988@hotmail.com 


\section{INTRODUCCIÓN}

El mecanismo por el cual el estrés calórico afecta negativamente la reproducción del ganado es probablemente multifactorial y puede variar dependiendo de la magnitud de este tipo de estrés. En vaquillas lecheras el estrés calórico reduce la longitud del estro y disminuye la expresión del mismo (Nebel y col 1997). Estos cambios en la actividad del estro causados por el estrés calórico reducen la probabilidad de detectar el celo (Hansen y Aréchiga 1999). Por otra parte, el estrés calórico también disminuye la fertilidad y la sobrevivencia embrionaria. Debido a lo anterior, algunos productores usan la monta natural durante el verano, como un medio para tratar de eliminar la necesidad de detectar celo o bien, adquieren semen de bajo costo, con la consecuente disminución del mérito genético del rebaño. El uso de la inseminación artificial a tiempo fijo (IATF) ofrece ventajas como la eliminación de la necesidad de la detección del estro lo cual es muy importante durante periodos de estrés calórico (Aréchiga y col 1998). No obstante lo anterior, el uso de IATF no protege al embrión de las altas temperaturas, por lo que se recomienda el manejo medio ambiental a través del uso de sistemas de enfriamiento (De la Sota y col 1998). Algunos estudios han reportado que el enfriamiento artificial por periodos cortos de tiempo ha tenido un alto (Gauthier 1983), pequeño (Stott y Wiersma 1976) o nulo (Her y col 1988) beneficio sobre la tasa de preñez en ganado lechero. Por otra parte, los bajos niveles de progesterona $\left(\mathrm{P}_{4}\right)$ observados en verano también pueden afectar la tasa de concepción debido a que esta tiene una importante función en la estimulación de algunas proteínas y factores de crecimiento indispensables para el desarrollo de la preñez (Geisert y col 1992). La suplementación con $\mathrm{P}_{4}$ ha incrementado la tasa de concepción cuando esta ha sido administrada al día 6 post IA (Mann y Lamming 1999).

En base a lo anterior, el objetivo de la presente investigación fue evaluar el efecto de la suplementación de $\mathrm{P}_{4}$ más un periodo corto de enfriamiento artificial post-IA sobre la respuesta fisiológica, hormonal y tasa de concepción de vaquillas Holstein durante el verano.

\section{MATERIAL Y MÉTODOS}

\section{UBICACIÓN DEL ÁREA DE ESTUDIO}

El experimento se llevó a cabo durante los meses de Julio a Agosto en una recría de vaquillas Holstein localizada en la carretera Mexicali-San Felipe Km.15, Municipio de Mexicali, B.C. La zona cuenta con un clima en verano caracterizado por ser cálido y seco, con una temperatura media anual de $22{ }^{\circ} \mathrm{C}$, en tanto que las máximas y mínimas promedian $48,5^{\circ} \mathrm{C}$ y $2^{\circ} \mathrm{C}$, respectivamente. La humedad relativa promedia durante los meses de verano $40 \%$ y la precipitación media anual de la zona es de 85 $\mathrm{mm}$. El THI mínimo promedia 74 alcanzando promedios máximos de 85 durante la época de verano (García 1985).

\section{ANIMALES EXPERIMENTALES Y TRATAMIENTOS}

Ciento treinta y siete vaquillas Holstein de 12 a13 meses de edad, con un peso promedio de $350 \pm 24 \mathrm{Kg}$, fueron agrupadas en uno de tres tratamientos. Tratamiento testigo $\left(\mathrm{T}_{1}\right)$, en el cual las vaquillas contaron únicamente con sombra en corral y un programa de IA (Inseminación Artificial) basado en detección visual de celo natural e IA de acuerdo a la regla AM-PM $(n=45)$. Un segundo tratamiento $\left(T_{2}\right)$, en donde las vaquillas estuvieron sujetas a un programa de IA al igual que en $T_{1}$, pero beneficiadas con un sistema de enfriamiento instalado bajo la sombra del corral y el cual proporcionó enfriamiento a las vaquillas desde el inicio del experimento hasta 21 d después de su IA $(n=46)$. El tercer tratamiento $\left(\mathrm{T}_{3}\right)$, consistió en vaquillas bajo un programa de inseminación artificial a tiempo fijo (IATF) como se muestra en la figura 1, más suplementación de progesterona del día 4 al 14 post-IA, mediante la reutilización

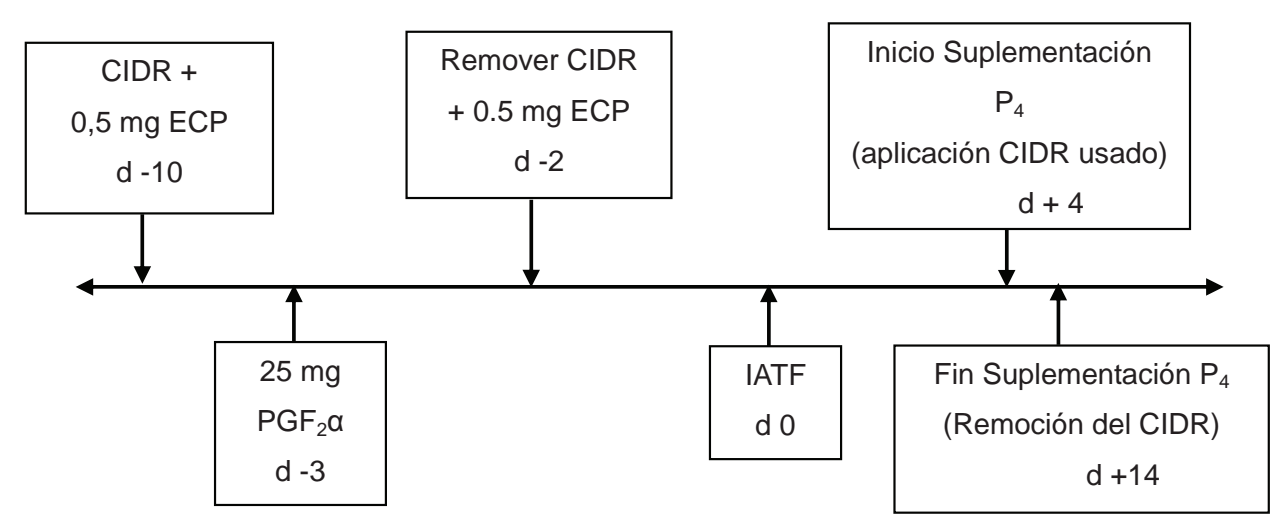

Figura 1. Protocolo de inseminación artificial a tiempo fijo y suplementación de progesterona aplicada a vaquillas Holstein (CIDR = Dispositivo intravaginal de liberación $\mathrm{de}_{4} ; \mathrm{ECP}=$ Cipionato de estradiol, Pfizer Animal Health; $\mathrm{PGF}_{2} \alpha=$ Lutalize, Pfizer Animal Health).

Timed artificial insemination protocol and progesterone supplementation applied to Holstein heifers. 
del dispositivo vaginal (CIDR) previamente utilizado en el protocolo de IATF. Este tratamiento estuvo sujeto a un enfriamiento artificial igual al proporcionado a $T_{2}(n=46)$.

Previo al inicio del experimento, a todas las vaquillas se les tomaron dos muestras de sangre con un intervalo de $10 \mathrm{~d}$, con el propósito de determinar los niveles de $\mathrm{P}_{4}$ y de esta manera identificar las vaquillas que se encontraban ciclando o no ciclando. Vaquillas con niveles mayores de $1 \mathrm{ng} / \mathrm{ml}$ de $\mathrm{P}_{4}$ en sangre en al menos un muestreo fueron consideradas como animales ciclando (Rivera y col 2005).

\section{INSTALACIONES EXPERIMENTALES}

Los animales fueron alojados en corrales abiertos con cerco de tubo de acero, proporcionando un espacio de $22 \mathrm{~m}^{2}$ por vaquilla. El área de sombra se ubicó en la parte oeste del corral, construida de lámina galvanizada y estructuras de tubo de acero, con orientación norte a sur. El espacio de sombra disponible fue de $2,3 \mathrm{~m}^{2}$ por vaquilla. En la parte norte de los corrales se localizó el área de comedero equipada con trampas individuales para facilitar el manejo de los animales. El comedero contaba con piso de concreto de una anchura de $2,1 \mathrm{~m}$, a partir de la pared del comedero.

\section{SISTEMA DE ENFRIAMIENTO}

El sistema de enfriamiento consistió en 6 ventiladores de $90 \mathrm{~cm}$ de diámetro, motor de $0,5 \mathrm{Hp}$, con una separación de $3 \mathrm{~m}$ entre uno y otro, y una producción de flujo de aire de $180 \mathrm{~m}^{3} / \mathrm{min} / \mathrm{ventilador}$. En cada uno de los ventiladores se instalaron 2 aspersores de agua con un gasto de $52 \mathrm{~L} / \mathrm{h}$. Los ventiladores fueron instalados bajo la sombra y operaron diariamente de las 9:30 a 18:30 h.

\section{ALIMENTACIÓN}

La alimentación de los animales fue ad libitum y consistió en una ración integral, la cual fue proporcionada diariamente a las 06:00, 09:00 y 18:00 h. La energía metaboliza- ble de la dieta fue $1,88 \mathrm{Mcal} / \mathrm{Kg}$ y los ingredientes y composición química de la misma se muestran en el cuadro 1.

\section{INFORMACIÓN CLIMATOLÓGICA}

La temperatura y humedad ambiental fueron registradas cada hora en la estación meteorológica de la UABC (Universidad Autónoma de Baja California) localizada a $15 \mathrm{~km}$ de distancia de la ubicación del experimento. La información recabada se utilizó para estimar el índice de temperatura-humedad (THI), mediante la fórmula propuesta por Hahn (1999).

$$
\mathrm{THI}=0,8 \mathrm{Ta}+\mathrm{HR}(\mathrm{Ta}-14,4)+46,4
$$

Donde: $\mathrm{THI}=$ Índice de temperatura-humedad

$\mathrm{Ta}=$ Temperatura ambiental $\left({ }^{\circ} \mathrm{C}\right)$

$\mathrm{HR}=\%$ Humedad relativa (expresada en decimales)

\section{VARIABLES EVALUADAS}

Las variables reproductivas evaluadas fueron: Tasa de concepción a primer servicio (TC), definida como el porcentaje de vaquillas que concibieron al primer servicio de IA. Días al primer servicio (DPS), estimado como el periodo de tiempo de cuando las vaquillas iniciaron en el experimento hasta que fueron inseminadas por primera vez. Días a la preñez (DP), considerado como el intervalo de tiempo entre el inicio de las vaquillas en el estudio hasta el servicio en que fueron diagnosticadas preñadas.

Las variables fisiológicas registradas fueron temperatura rectal (TR) a las 18:00 h, recabada por medio de un termómetro digital (Fluke 51 II, USA) en todas las vaquillas de cada tratamiento; en tanto que la frecuencia respiratoria (FR), fue medida en 5 vaquillas por tratamiento a las 17:00 h contando los movimientos del flanco durante un minuto. Ambas mediciones fueron registradas tres veces por semana (lunes, miércoles, viernes).

Así mismo se colectaron temperaturas vaginales (TV) de 3 vaquillas por tratamiento utilizando un sensor

Cuadro 1. Ingredientes y composición química de la dieta.

Ingredients and chemical composition of the diet.

\begin{tabular}{lclc}
\hline Ingrediente & $\%$ & Composición química & $\%$ \\
\hline Sorgo grano & 20 & Materia seca & 83,3 \\
Bermuda & 11 & Humedad & 16,6 \\
Alfalfa-cebada & 16 & Proteína cruda & 9,2 \\
Sudan & 12 & Extracto etéreo & 4,5 \\
Gluten de maíz & 8 & Cenizas & 11,1 \\
Semilla de algodón & 5 & FDN & 47,3 \\
Melaza & 10 & & \\
Premix vaquilla & 2 & & \\
\hline
\end{tabular}


HOBO U12 sujetado a un dispositivo CIDR (sin progesterona) y colocado en la vagina durante $24 \mathrm{~h}$. El sensor registró la temperatura vaginal cada 15 min en martes y jueves de cada semana. Adicionalmente se colectaron muestras sanguíneas los días 5, 9, 14 post-IA para determinar la concentración de progesterona. Una vez colectadas las muestras de sangre, fueron conservadas en hielo y llevadas al laboratorio para ser centrifugadas a 3000 rpm durante 15 minutos y almacenadas a $-20{ }^{\circ} \mathrm{C}$, para su posterior análisis en el laboratorio de la Facultad de Medicina de la UABC por medio de la prueba de Elisa, utilizando un kit para progesterona validado comercialmente (Bio Check, Inc, Foster City, Ca).

\section{ANÁLISIS ESTADÍSTICO}

La tasa de concepción fue analizada por medio de una prueba de chi-cuadrado utilizando el procedimiento PROC FREQ. La frecuencia respiratoria, temperatura vaginal y rectal así, como la concentración de progesterona, se analizaron a través de un modelo de medidas repetidas en el tiempo mediante PROC MIXED. Las variables días a primer servicio y días a la preñez fueron sometidas aun análisis de varianza con el procedimiento PROC GLM utilizando un diseño completamente al azar. No obstante que las variables de respuesta (frecuencia respiratoria, temperatura rectal, días a primer servicio y días a la concepción) fueron variables continuas, se sometieron a un análisis Shapiro-Wilk para confirmar su distribución normal (Freund 1992), por lo que no requirieron de transformación alguna. El nivel de significancia fue del $5 \%$ y la diferencia detectada entre 5 y $10 \%$ se consideró como tendencia. Todos los procedimientos estadísticos utilizados se desarrollaron con el paquete estadístico SAS (2004) versión 9.12 para Windows.

\section{RESULTADOS}

\section{CONDICIONES CLIMATOLÓGICAS}

Las variables climatológicas registradas durante el estudio fueron divididas en período de día (06:00 a 17:00 h) y de noche (18:00 a 05:00 h). De acuerdo a Wiersma (1990), los valores de THI registrados indicaron que los animales experimentales estuvieron bajo un estrés térmico de medio a severo en la mayor parte de las horas del día (cuadro 2).

\section{RESPUESTA FISIOLÓGICA, HORMONAL Y EFICIENCIA REPRODUCTIVA}

La frecuencia respiratoria fue reducida en $10 \%$ y $7 \%$ para $\mathrm{T}_{2}$ y $\mathrm{T}_{3}$ respectivamente con respecto al grupo testigo $(\mathrm{P}<0,05)$. En tanto, la TR no mostró una disminución significativa $(\mathrm{P}>0,05)$ en respuesta al enfriamiento. La tasa de concepción obtenida fue similar $(\mathrm{P}>0,05)$ para los 3 tratamientos. Sin embargo, las vaquillas en $\mathrm{T}_{3}$ requirieron de menos días $(\mathrm{P}<0,05)$ para su primer servicio en comparación con $\mathrm{T}_{2} \mathrm{y}$ un similar número de días $(\mathrm{P}>$ $0,05)$ comparado con $T_{1}$ No obstante, los días a la preñez fueron similares $(\mathrm{P}>0,05)$ entre tratamientos (cuadro 3 ). Los niveles de $\mathrm{P}_{4}$ durante la fase lútea no fueron incrementados $(\mathrm{P}>0,05)$ con la suplementación de $\mathrm{P}_{4}$ en ninguno de los días de muestreo mediante la inserción vaginal del CIDR reciclado del día 4 al 14 post IA (figura 2).

\section{TEMPERATURA VAGINAL}

Las temperaturas vaginales registradas durante las 24 horas del día sólo difirieron $(\mathrm{P}<0,05)$ entre el grupo testigo y los tratamientos $\mathrm{T}_{2}$ y $\mathrm{T}_{3}$ de las 10:00 a las 17:00, en tanto que desde las 18:00 a las 9:00 h la TV fue similar $(P>0,05)$ entre los 3 tratamientos, lo cual demostró que los animales del grupo testigo a los cuales no se les proporcionó enfriamiento pudieron mantener su temperatura a niveles normales cercanos a $\operatorname{los} 38,5^{\circ} \mathrm{C}$ durante las horas de la noche (figura 3 ).

\section{DISCUSIÓN}

Los índices de temperatura y humedad que se registraron durante el período de la prueba indicaron que las vaquillas estuvieron sujetas a un estrés térmico tanto durante el día como la noche. Sin embargo, las temperaturas vaginales de las vaquillas en los 3 tratamientos estuvieron por debajo de los $39^{\circ} \mathrm{C}$ por aproximadamente $6 \mathrm{~h}$ (01:00 - 07:00 h), lo cual demostró que las vaquillas tuvieron el tiempo y las condiciones climáticas necesarias para la recuperación, pudiendo mantener su temperatura vaginal a niveles normales durante las horas de la noche. Igono y col (1992) indicaron que valores por debajo de $39^{\circ} \mathrm{C}$ son considerados normales de temperatura corporal en ganado lechero.

El índice de temperatura y humedad actualmente disponible no es buen indicador del nivel de estrés térmico, ya que considera solamente la temperatura y humedad (Mader y col 2006), no obstante, se ha reconocido que la velocidad del viento y la radiación solar también alteran la habilidad del animal para mantener su balance térmico (Brosh y col 1998). En el presente estudio, aún y cuando el THI fue alto durante las horas de la noche, probablemente la reducción de la radiación solar y un incremento de la velocidad del viento durante la noche permitieron que los animales disminuyeran su temperatura corporal en ese lapso de tiempo, reduciendo con ello el impacto negativo del estrés térmico sobre la reproducción. Lo anterior sugiere que los estudios relacionados con estrés calórico deben considerar analizar variables climatológicas como temperatura ambiente, humedad relativa, radiación solar, y velocidad de viento no sólo durante las horas del día, que son las más cálidas si no también durante las horas de la noche, las cuales finalmente vendrán a de- 
Cuadro 2. Promedios de temperatura ambiental, humedad relativa e índice temperatura-humedad (THI) registrados durante el periodo experimental.

Average values for ambient temperature, relative humidity and temperature-humidity index (THI) recorded during the experimental period.

\begin{tabular}{lcccc}
\hline \multicolumn{1}{c}{ Variable } & Día $^{\mathrm{a}}$ & Noche $^{\mathrm{b}}$ & Máximo & Mínimo \\
\hline Temperatura ambiental $\left({ }^{\circ} \mathrm{C}\right)$ & 37,7 & 32,4 & 42,8 & 28,1 \\
Humedad relativa $(\%)$ & 31,9 & 44,0 & 61,3 & 17,3 \\
THI & 84,0 & 80,0 & 86,0 & 78,0 \\
\hline
\end{tabular}

${ }^{a} 06: 00-17: 00 \mathrm{~h}$

${ }^{\mathrm{b}} 18: 00-05: 00 \mathrm{~h}$

Cuadro 3. Respuesta fisiológica y eficiencia reproductiva de vaquillas Holstein suplementadas con progesterona y enfriamiento artificial post IA en verano (Media \pm DE).

Physiological responses and reproductive performance of Holstein heifers supplemented with progesterone and artificial cooling post AI in summer $($ Mean $\pm \mathrm{SD})$.

\begin{tabular}{|c|c|c|c|c|c|}
\hline Tratamiento & $\mathrm{FR}^{1}$ & $\mathrm{TR},{ }^{\circ} \mathrm{C}$ & $\mathrm{TC}, \%$ & DPS & DP \\
\hline $\mathrm{T}_{1}$ & $89,0 \pm 6,5^{\mathrm{a}}$ & $40,0 \pm 0,57^{\mathrm{a}}$ & $53,3^{\mathrm{a}}$ & $13,0 \pm 2,1^{\mathrm{ab}}$ & $39,0 \pm 13,4^{a}$ \\
\hline $\mathrm{T}_{2}$ & $80,0 \pm 6,5^{\mathrm{b}}$ & $39,8 \pm 0,57^{\mathrm{a}}$ & $58,7^{\mathrm{a}}$ & $14,0 \pm 2,1^{\mathrm{a}}$ & $42,0 \pm 13,4^{a}$ \\
\hline $\mathrm{T}_{3}$ & $83,0 \pm 6,5^{\mathrm{c}}$ & $39,8 \pm 0,57^{\mathrm{a}}$ & $47,8^{\mathrm{a}}$ & $10,0 \pm 2,1^{\mathrm{b}}$ & $39,0 \pm 13,4^{a}$ \\
\hline
\end{tabular}

${ }^{\mathrm{a}, \mathrm{b}}$ Medias dentro de una misma columna con distinta literal difieren entre si $(\mathrm{P}<0,05)$.

$\mathrm{FR}=$ Frecuencia respiratoria, $\mathrm{TR}=$ Temperatura rectal, $\mathrm{TC}=$ Tasa de concepción, $\mathrm{DPS}=$ Días a primer servicio, $\mathrm{DP}=\mathrm{Días}$ a la preñez, $\mathrm{DE}=\mathrm{Desviación}$ estándar.

${ }^{1}$ Expresada como respiraciones por minuto.

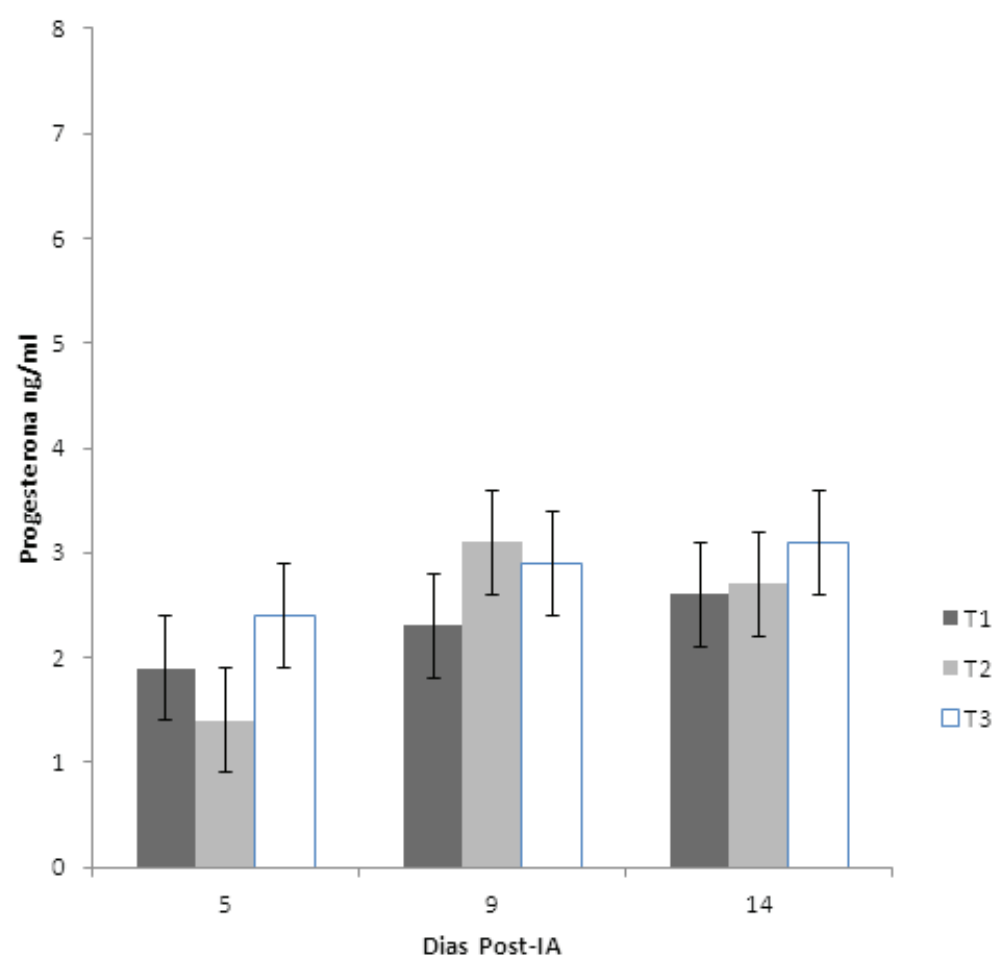

Figure 2. Concentración de progesterona en suero a diferentes días post-IA ( de muestreo).

Progesterone concentration in serum to different days post-AI (no significant differences $(\mathrm{P}>0.05)$ into days of sampling). 


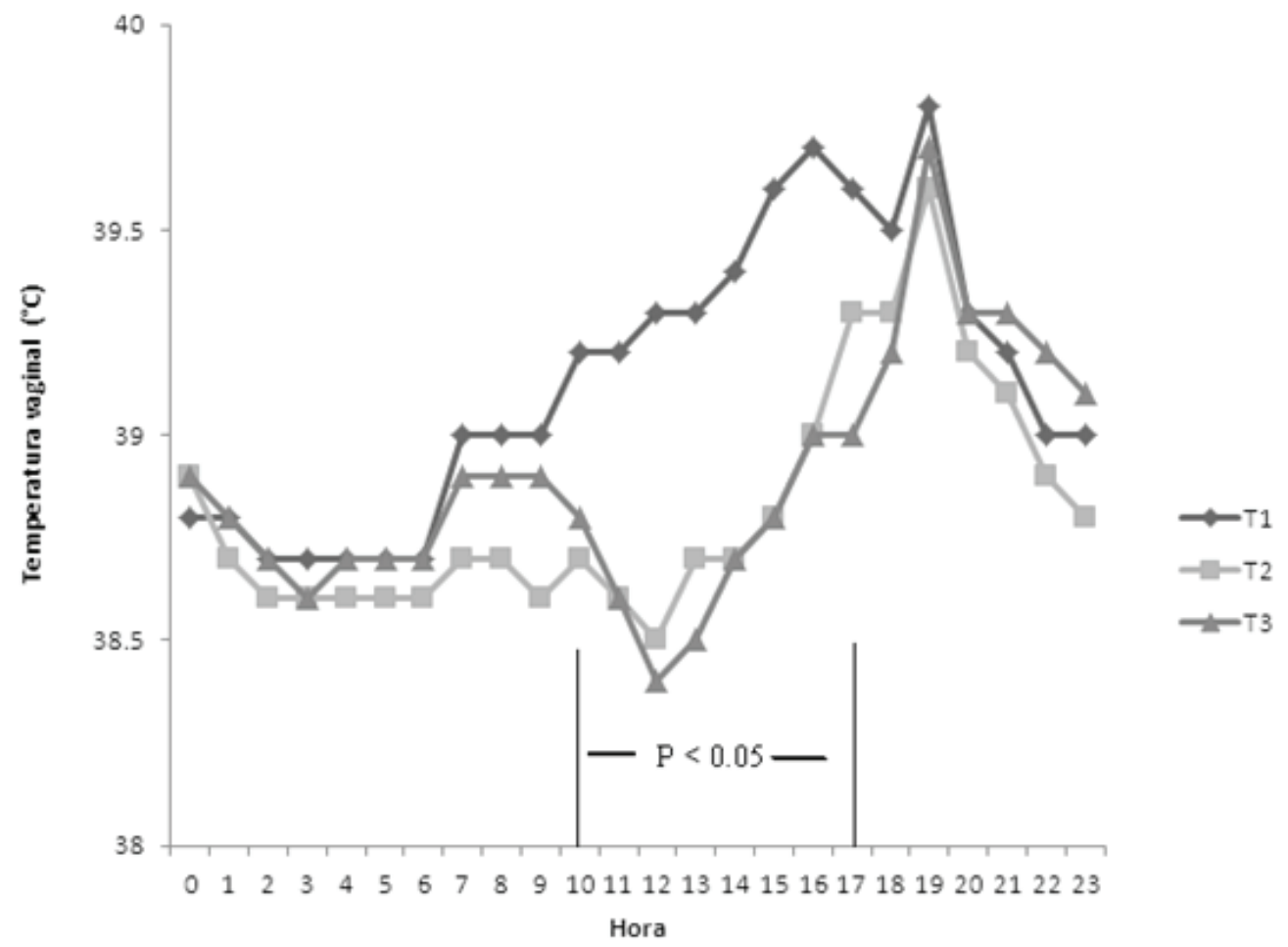

Figura 3. Temperatura vaginal durante las 24 horas del día. Diferencias significativas $(\mathrm{P}<0,05)$ solo de 10:00 a 17:00 h. Vaginal temperature during 24 hours. Significant difference $(\mathrm{P}<0.05)$ only from 10:00 to 17:00 h.

terminar el impacto del estrés calórico sobre la eficiencia productiva y reproductiva del ganado lechero (Shishido y col 1983, Correa y col 2000).

La frecuencia respiratoria registrada a las 17:00 h fue reducida en alrededor de un $9 \%$ en los tratamientos bajo enfriamiento comparado a $\mathrm{T}_{1}$, mientras que la temperatura rectal recabada a las 18:00 h fue reducida en $0,2{ }^{\circ} \mathrm{C}$. Sin embargo, estas reducciones están por debajo de lo reportado por otros estudios de reducciones de $18 \%$ en FR y $0,4^{\circ} \mathrm{C}$ en TR, en la misma zona y con el uso del mismo sistema de enfriamiento (Correa-Calderón y col 2009a, Correa-Calderón y col 2009'). Otros investigadores también han reportado reducciones de la temperatura rectal entre 0,4 a $0,5{ }^{\circ} \mathrm{C}$ al proporcionar enfriamiento mediante aspersores y ventiladores (Bucklin y col 1991), y reducciones de $15 \%$ en la frecuencia respiratoria al asperjar agua sobre vaquillas en verano (Marcillac-Embertson y col 2009). Durante el desarrollo del estudio se observó un incremento en la actividad física, principalmente en el área de comederos de algunas vaquillas en los tratamientos bajo enfriamiento, ya que permanecieron fuera del área de sombra y enfriamiento por diferentes espacios de tiempo a partir de las 16:00 hasta las 18:00 h, probablemente ocasionado por el confort proporcionado por el tratamiento de enfriamiento, lo que estimuló el apetito, ya que este comportamiento no se observó en el tratamiento testigo. Lo anterior pudo provocar que estas vaquillas fuera del área de sombra tuvieran una mayor car- ga de calor al momento de registrar la temperatura rectal a las 18:00 h, explicando el por qué no se observó una diferencia entre tratamientos en esta variable. En general, los resultados de las variables fisiológicas en el presente estudio mostraron un alivio al estrés calórico por parte de las vaquillas bajo enfriamiento como fue indicado por la FR y TV. El incrementar la circulación de aire por medio de ventiladores aumenta el enfriamiento, sobre todo en vaquillas bajo aspersión de agua, lo que trae consigo una significativa pérdida de calor corporal.

Es muy posible que una significante proporción de la reducción de la fertilidad en la población de vacas sea atribuible a una falla en la función lútea materna y consecuentemente un inadecuado ambiente uterino dependiente de la progesterona (Wiebold 1988). Diversos estudios han mostrado resultados controversiales en cuanto al efecto del estrés calórico sobre los niveles de progesterona durante el diestro, ya que algunos han reportado incrementos (Vaught y col 1977), reducciones (Wolfenson y col 1988) o ningún efecto (Wise y col 1988), como fue el caso del presente estudio, aún en el tratamiento donde se suplementó $\mathrm{P}_{4}$. En este sentido se ha señalado que la intensidad y duración del estrés calórico, edad del animal, etapa de la lactancia y alimentación son factores que pueden contribuir a la variación de los niveles de progesterona plasmática durante el verano (Trout y col 1998). Asimismo, Lima y col (2009) reportaron que ni la suplementación de progesterona, ni la cantidad de la 
misma hormona suplementada, beneficiaron la fertilidad de vacas lecheras sujetas a IATF. Estos mismos autores reportan que los incrementos de progesterona fueron 0,9 $\mathrm{ng} / \mathrm{ml}$ por CIDR utilizado y que se pueden requerir de incrementos mayores de 1,8 ng/ml (2 CIDR) cuando progesterona exógena es suplementada para observar un beneficio sobre la tasa de preñez, principalmente en vacas con bajos niveles de progesterona $(<1 \mathrm{ng} / \mathrm{ml})$ al inicio del protocolo de IATF. Resultados de estudios sobre los efectos de incorporar dispositivos vaginales conteniendo progesterona (CIDR) en vacas bajo IATF para incrementar fertilidad han sido inconsistentes y esto es atribuido a diferencias en la genética, en producción de leche, tipo de protocolo de IATF usado y concentración de progesterona en la vaca durante el tratamiento con CIDR (Gümen y Wiltbank 2005). Por otra parte, El-Zarkouny y Stevenson (2004) no detectaron diferencias en las concentraciones de progesterona en sangre con la inserción de CIDR nuevos o usados del día 13 al 20 después de la IATF. Estos autores atribuyen la falta de diferencias en los niveles de progesterona a una baja concentración residual de $\mathrm{P}_{4}$ en el CIDR usado, además que los CIDR nuevos sólo incrementan la $\mathrm{P}_{4}$ marginalmente comparado con los cambios durante la fase luteal o durante la preñez. Resultados similares han sido reportados por otros estudios donde la suplementación de $\mathrm{P}_{4}$ por medio de CIDR no incrementó los niveles de esta hormona en vacas y vaquillas lecheras al principio de la gestación (día 4 a 18 post-IA) (Wendy y col 2009). Sin embargo, en una investigación reciente se logró incrementar los niveles de progesterona con la aplicación de CIDR del día 5 al 19 post-IA, observándose también un incremento en la tasa de preñez en comparación a un grupo testigo (Mehni y col 2012).

La tasa de concepción alcanzada por el tratamiento testigo se encontró por arriba del $30 \%$ reportado en trabajos previos en la misma zona de estudio (CorreaCalderón y col 2009a ; Correa-Calderón y col 2009ªb. La reducción de la temperatura vaginal en horas tempranas de la mañana hace suponer que el estrés térmico en el verano en que se desarrolló la prueba pudo no afectar al ganado tan drásticamente como en otros años. Un estudio conducido en Florida por Badinga y col (1985) mostró que vaquillas vírgenes tuvieron una tasa de concepción de $50 \%$ durante el verano y sufrieron solo una ligera disminución en su fertilidad en esa época del año. Este mismo estudio señala que algunos otros factores como hora, número de inseminaciones, inseminador y el año de servicio pueden afectar la fertilidad y no sólo las altas temperaturas ambientales.

La suplementación de $\mathrm{P}_{4}$ y enfriamiento artificial postIATF no mejoró la tasa de concepción, sin embargo, la mantuvo similar a vaquillas inseminadas a celo natural, lo que no se ha observado en otros estudios. Es posible que la cantidad de $\mathrm{P}_{4}$ proporcionada por el CIDR usado no fuera suficiente para incrementar los niveles de esta hormona durante la fase luteal. Los registros de TV durante la noche señalan que las vaquillas del grupo testigo lograron perder suficiente carga de calor como para mantener niveles adecuados de TV similares a los grupos enfriados.

\section{AGRADECIMIENTOS}

La investigación fue apoyada con recursos de la Universidad Autónoma de Baja California. Se agradece en forma especial al Ing. Juan Carlos Reynoso Maldonado, propietario, y al Ing. Rubén Fregoso Cardoso, encargado de Corrales San Carlos, por permitir el uso de sus animales e instalaciones.

\section{REFERENCIAS}

Aréchiga CF, CR Staples, LR McDowell, PJ Hansen.1998. Effects of timed insemination and supplemental $\beta$-carotene on reproduction and milk yield of dairy cows under heat stress. J Dairy Sci 81, 390-402.

Badinga L, RJ Collier, WW Thatcher, CJ Wilcox. 1985. Effect of climatic and management factors on conception rate of dairy cattle in subtropical environment. J Dairy Sci 68, 78-85

Brosh A, A Aharoni, A Degen, D Wright, B Young. 1998. Effects of solar radiation, dietary energy, and time of feeding on thermoregulatory responses and energy balance in cattle in hot environment. J Anim Sci 76, 2671-2677.

Bucklin RA, LW Turner, DK Beede, DR Bray, RW Hemkem. 1991. Methods to relieve heat stress for dairy cows in hot, humid climates. Appl Eng Agric 7, 241-247.

Correa A, DV Armstrong, DE Ray, RM Enns, CM Howison, HG González, FJ Verdugo, AP Márquez. 2000. Correlation coefficients among productive, physiological, and hormonal responses and temperature-humidity index in heat stressed dairy cows. J Dairy Sci 83 (Suppl.1), 34.

Correa-Calderón A, G Des Santos, L Avendaño, F Rivera, D Alvarez, F Ardon, R Diaz, R Collier. 2009a. Enfriamiento artificial y tasa de concepción de vaquillas Holstein con estrés térmico. Arch Zootec 58, 231-239.

Correa-Calderón A, C Leyva, L Avendaño, F Rivera, R Diaz,

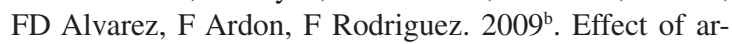
tificial cooling and its combination with timed artificial insemination on fertility of Holstein heifers during the summer. J Appl Anim Res 35, 109-112.

De la Sota RL, JM Burke, CA Risco, F Moreira, MA De Lorenzo, WW Thatcher. 1998. Evaluation of timed insemination during summer heat stress in lactating dairy cattle. Theriogenology 49, 761-770.

El-Zarkouni SZ, JS Stevenson. 2004. Resynchronizing estrus with progesterone or progesterone plus estrogen in cows of unknown pregnancy status. J Dairy Sci 87, 3306-3321.

Freund JE. 1992. Mathematical Statistics. Prentice-Hall, New Jersey, USA.

García E. 1985. Modificaciones al sistema de clasificación de Köppen (para adaptarlo a las condiciones de la República Mexicana). 2a ed. Instituto de Geografía, Universidad Nacional Autónoma de México, México, D.F, México.

Gauthier D.1983. A technique for improving the fertility of French Friesian cows in a tropical climate. Effect on plasma progesterone profile. Reprod Nutr Dev 23, 129-136.

Geisert RD, GL Morgan, EC Short, MT Savy. 1992. Endocrine 
events associated with endometrial function and conceptus development in cattle. Reprod Fertil Dev 4, 301-305.

Gümen A, MC Wiltbank. 2005. Length of progesterone exposure needed to resolve large follicle anovular condition in dairy cows. Theriogenology 63, 202-218.

Hahn GL. 1999. Dynamic responses of cattle to thermal heat loads. J Anim Sci 77 (Suppl. 2), 10-20.

Hansen PJ, CF Aréchiga. 1999. Strategies for managing reproduction in heat-stressed dairy cow. J Dairy Sci 82 (Suppl. 2), 36-50.

Her E, D Wolfenson, I Flamenbaun, I Folman, M Kaim, A Berman. 1988. Thermal, productive, and reproductive responses of high yielding cows exposed to short-term cooling in summer. J Dairy Sci 71, 1085-1092.

Igono MO, G Bjotvedt, HT Sanford-Crane. 1992. Environmental profile and critical temperature effects on milk production of Holstein cows in desert climate. Int J Biometeorol 36, 77-87.

Lima JR, FA Rivera, CD Narciso, R Oliveira, RC Chebel, JEP Santos. 2009. Effect of increasing amounts of supplemental progesterone in a timed artificial insemination protocol on fertility of lactating dairy cows. J Dairy Sci 92, 5436-5446.

Mader TL, MS Davis, T Brown-Brandl. 2006. Environmental factors influencing heat stress in feedlot cattle. J Anim Sci 84, 712-719.

Mann GE, GE Lamming. 1999. The influence of progesterone during early pregnancy in cattle. Reprod Domest Anim 34, 269-274.

Marcillac-Embertson NM, PH Robinson, JG Fadel, FM Mitloehner. 2009. Effects of shade and sprinklers on performance, behavior, physiology, and the environment of heifers. J Dairy Sci 92, 506-517.

Mehni SB, HK Shabankareh, M Kazemi-Bonchenari, M Eghbali. 2012. The Comparison of treating Holstein dairy cows with progesterone, CIDR and GnRH after insemination on serum progesterone and pregnancy rates. Reprod Domest Anim 47, 131-134.

Nebel RL, SM Jobst, MBG Dransfield, SM Pandolfi, TL Bailey.
1997. Use of radiofrequency data communication system, Heat Watch ${ }^{\circledR}$ to describe behavioral estrus in dairy cattle. J Dairy Sci 80 (Suppl. 1), 179.

Rivera H, Lopez H, Fricke PM. 2005. Use of intravaginal progesterone-releasing inserts in a synchronization protocol before timed $\mathrm{AI}$ and for synchronizing return to estrus in Holstein heifers. J Dairy Sci 88, 957-968.

SAS INSTITUTE. 2004. SAS/STAT: user's guide statistics released 9. $12^{\text {th }}$ ed. SAS Institute. Inc., Cary, NC., USA.

Shishido H, N Yamagishi, T Mitsuhashi. 1983. Effect of diurnal temperature cycle on milk performance of Holstein cows. Proc $5^{\text {th }}$ Wld Conf Anim Prod 2, 785-786.

Stott GH, F Wiersma. 1976. Short term thermal relief for improved fertility in dairy cattle during hot weather. Int $J$ Biometeorol 20, 344-350.

Trout JP, LR McDowell, PJ Hansen. 1998. Characteristics of the estrous cycle and antioxidant status of lactating Holstein cows exposed to heat stress. J Dairy Sci 81, 1244-1250.

Vaught LW, DE Monty, WC Foote.1977. Effect of summer heat stress on serum luteinizing hormone and progesterone values in Holstein-Friesian cows in Arizona. Am J Vet Res 38, 1027-1030.

Wendy JA, J Andrew, JA Holle, ML Bauer, JD Kirsch, DE Schimek, KG Odde, KA Vonnahme. 2009. Effect of postinsemination progesterone supplementation on pregnancy rate in dairy cows. Can J Vet Res 73, 271-274.

Wiebold JL. 1988. Embryonic mortality and the uterine environment in first service lactating dairy cows. J Reprod Fertil 84, 393-399.

Wiersma F. 1990. THI modified for dairy cows. Department of Agricultural Engineering. The University of Arizona. Tucson, Arizona, USA.

Wise ME, DV Armstrong DV, JT Huber, R Hunter, F Wiersma.1988. Hormonal alterations in the lactating dairy cow in response to thermal stress. J Dairy Sci 71, 2480-2485.

Wolfenson D, I Flamenbaum, A Berman. 1988. Hyphertermia and body energy store effects on estrus behavior, conception rate, and corpus luteum function in dairy cows. $J$ Dairy Sci 71, 3497-3504. 